\title{
MANFRED LÖWISCH
}

\author{
Die Anpassung der Arbeitsentgelte an die \\ Insolvenzsituation de lege lata und de lege ferenda
}




\title{
Die Anpassung der Arbeitsentgelte an die Insolvenzsituation de lege lata und de lege ferenda
}

\author{
von \\ Professor Dr. Manfred Löwisch, Freiburg i. Breisgau
}

\section{Inbaltsübersicht}

A. Die Anpassung der Arbeitsentgelte de lege lata

I. Die Anpassung tariflich festgelegter Entgelte

II. Die Anpassung in Betriebsvereinbarungen festgelegter Entgelte

III. Die Anpassung einzelvertraglich festgelegter Entgelte

B. Die Anpassung der Arbeitsentgelte de lege ferenda

I. Die Anpassung nicht tariflich festgelegter Entgelte

II. Die Anpassung tariflich festgelegter Arbeitsentgelte

\section{A. Die Anpassung der Arbeitsentgelte de lege lata}

\section{Die Anpassung tariflich festgelegter Entgelte}

1. These: Tariflich festgelegte Arbeitgeberleistungen können durch Vereinbarung eines neuen, den bisherigen Tarifvertrag ablösenden Tarifvertrags an die Insolvenzsituation angepaßt werden.

Daß tarifvertraglich festgelegte Ansprüche der Arbeitnehmer durch einen den bisherigen Tarifvertrag ablösenden Tarifvertrag herabgesetzt werden können, ist nicht zweifelhaft. Als Rechtsnormen werden die Bestimmungen eines Tarifvertrages nicht selbst Inhalt der einzelnen Arbeitsverhältnisse, sondern bestimmen diesen nur, solange sie gelten. Sie können deshalb durch einen neuen Tarifvertrag jederzeit ersetzt werden ${ }^{1}$. Ein derartiger Tarifvertrag unterliegt auch keiner Billigkeitskontrolle. Lediglich der Vertrauensschutzgedanke zieht ihm äußerste Grenzen². Soweit es um künftige Entgelte geht, verlangt dieser nur, daß den Arbeitnehmern ausreichend Zeit gelassen wird, um sich auf die veränderte Situation einzustellen. In diesem Sinne für angemessene Übergangsfristen bei Einführung der Neuregelung zu sorgen, ist Sache der Tarifvertragsparteien.

1 Vgl. zuletzt BAG vom 14.12. 1082, DB 1983, 944.

2 BAG DB 1983, 944. 
2. These: Möglich ist dabei sowobl die Vereinbarung eines Werkstarifvertrages mit dem insolventen Unternehmen selbst als auch die Vereinbarung eines nur für dieses Unternebmen geltenden Verbandstarifvertrages.

Wenn die Arbeitsbedingungen in dem insolvent gewordenen Unternehmen bisher in einem Werkstarifvertrag geregelt waren, bietet die Ablösung dieses Werkstarifvertrages durch einen neuen Werkstarifvertrag keinerlei Probleme. Aber auch wenn das insolvent gewordene Unternehmen einem Arbeitgeberverband angehört und demzufolge einem Verbandstarifvertrag unterliegt, ist der Abschluß eines Werkstarifvertrages möglich. Die Zugehörigkeit zu einem Arbeitgeberverband ändert nichts an der nach $\$ 2$ Abs. 1 TVG gegebenen Tariffähigkeit auch als einzelner Arbeitgeber ${ }^{3}$. $\mathrm{Daß}$ der Abschluß des Werkstarifvertrages möglicherweise gegen die Mitgliedschaftspflichten des Unternehmers gegenüber dem Arbeitgeberverband verstößt und damit zu mitgliedschaftsrechtlichen Sanktionen führen $\mathrm{kann}^{32}$, ändert an der Wirksamkeit eines abgeschlossenen Werkstarifvertrages nichts.

Möglich ist auch der Abschluß eines lediglich für das insolvente Unternebmen geltenden Verbandstarifvertrages. Die Bedenken, die generell oder für bestimmte Konstellationen gegen sog. firmentarifbezogene Verbandstarifverträge vorgebracht werden, treffen den firmenbezogenen Verbandstarifvertrag, mit dem der Insolvenzsituation eines Unternehmens Rechnung getragen werden soll, durchweg nicht. Weder ist die Friedensfunktion des Tarifvertrages in Frage gestellt ${ }^{4}$. Noch liegt eine willkürliche Ungleichbehandlung des insolventen Unternehmens und damit ein Verstoß gegen Art. 3 GG vor. Noch auch wäre der verbandsrechtliche Gleichbehandlungsgrundsatz tangiert, an dem insbesondere Buchner und Richardi firmenbezogene Verbandstarifverträge messen wollen ${ }^{6}:$ Zwar steht nach dieser Auffassung die verbandsrechtliche Gleichbehandlungspflicht nicht nur einer Benachteiligung, sondern auch einer Bevorzugung einzelner Mitglieder entgegen. Aber ihre Vertreter räumen ein, daß die Herausnahme einzelner Arbeitgeber aus dem Geltungsbereich tariflicher Regelungen nicht notwendig auf Kosten anderer Mitglieder des Verbands geht. Erst wo die Herausnahme Einfluß auf das Ergebnis der Tarifverhandlungen für die übrigen Mitglieder hat, sehen sie einen Verstoß gegen die Gleichbehandlungspflicht

3 Allgemeine Ansicht, vgl. WIEdemann/StumpF, Komm. z. TVG, 5. Aufl. 1977, $\$ 2$ Anm. 82.

3 a Vgl. zu solchen Sanktionen für den Fall der Nichtbefolgung eines Aussperrungsbeschlusses Löwisch/KRAUSs, AR-Blattei, D-Blatt Arbeitskampf III unter B VIII.

4 Vgl. die diesbezüglichen Bedenken von MAYER-MALY, DB 1965, 32, 33.

5 Vgl. zu dieser Grenze Wiedemann/StumpF, aaO (Fn. 3), \$2 TVG Anm. 89.

6 BUCHNER, DB 1970, 2025, 2030; RICHARDI, Jura 1971, 141, 168 ff; vgl. auch DÄUBLER/ HEGE, Tarifvertragsrecht, 2. Aufl., 1981, Anm. $354 \mathrm{ff.}$ 
gegeben'. Davon aber kann bei der Herausnahme eines Unternehmens aus der Geltung des Verbandstarifvertrages wegen der Sondersituation der Insolvenz nicht gesprochen werden.

Während der für das insolvente Unternehmen abgeschlossene firmenbezogene Verbandstarifvertrag den bisherigen Verbandstarifvertrag in seiner Geltung für das Unternehmen ohne weiteres ablöst, entsteht bei dem Abschluß eines Werkstarifvertrages mit einem verbandsangehörigen Unternehmen das Problem der Tarifkonkurrenz. Sie ist nach dem Spezialitätsgrundsatz dahin zu lösen, daß der Werkstarifvertrag dem bisher geltenden Verbandstarifvertrag vorgeht ${ }^{8}$.

3.These: Die tarifliche Neuregelung in einem unternebmensbezogenen Verbandstarifvertrag kann auch in der Vereinbarung einer für das insolvente Unternebmen geltenden Öffnungsklausel gegenüber Betriebsvereinbarung oder Arbeitsvertrag besteben.

Wenn $\int 4$ Abs. 3 TVG von einem Tarifvertrag abweichende „Abmachungen“ dann zuläßt, wenn sie durch den Tarifvertrag gestattet sind, so meint er damit sowohl die Betriebsvereinbarung wie die arbeitsvertragliche Abrede. Eine entsprechende Öffnungsklausel kann auch nachträglich in einen Tarifvertrag aufgenommen werden.

4. These: Die Vereinbarung einer Anrecbnungsklausel, nach der auf die tariflichen Entgelte übertarifliche Entgelte angerechnet werden können, ist unzulässig.

Von Anfang an oder nachträglich vereinbarte Tarifvertragsklauseln, nach denen auf die tariflichen Entgelte übertarifliche Entgelte angerechnet werden können, sind rechtlich nicht wirksam, weil sie entgegen dem Prinzip des $\$ 4$ Abs.3 TVG tarifliche Höchstarbeitsbedingungen setzen'. Ob übertarifliche Entgelte auf tarifliche Entgelte angerechnet werden oder nicht, kann sich nur nach dem Arbeitsvertrag richten. Dieser kann im Einzelfall einmal dahin zu verstehen sein, daß übertarifliche Entgelte auf eine Tariflohnerhöhung angerechnet werden sollen. Dahin, daß eine Anrechnung nur deshalb möglich sein soll, weil die Tarifvertragsparteien eine solche Anrechnung in Vorschlag bringen, wird er aber kaum jemals zu verstehen sein.

5. These: Eine vereinbarte tarifliche Neuregelung gilt für nichttarifgebundene Arbeitsverhältnisse dann, wenn diese auf den Tarifvertrag Bezug nebmen.

7 Vgl. BuCHNER, DB 1970, 2025, 2030.

$8 \mathrm{Vgl}$. Wiedemann/STumpF, aaO (Fn. 3), \$4 TVG Anm. 165.

9 Vgl. BAG vom 18.8.1971, AP Nr.8 zu \4 TVG Effektivklausel $=$ DB 1971, $2117=$ BB 1971, 1366; Buchner, DB 1970, 2025, 2030; WIEDEMANN/STumpF, aaO (Fn. 3), $\$ 4$ TVG Anm. 271. 
An sich beschränkt sich die Geltung sowohl des bisherigen wie des diesen ändernden Tarifvertrages nach $\$ 3$ Abs. 1 TVG auf die beiderseitig Tarifgebundenen. Ist aber für die nicht tarifgebundenen Arbeitsverhältnisse, wie das häufig ist, auf die jeweils geltenden einschlägigen Tarifverträge Bezug genommen, so nehmen die betreffenden Arbeitsverhältnisse regelmäßig auch an der Änderung des bisher geltenden Tarifvertrages teil. Dies gilt auch, wenn der ändernde Tarifvertrag die Arbeitsbedingungen verschlechtert, solange diese Verschlechterung im Rahmen des Angemessenen bleibt ${ }^{10}$. Von solcher Angemessenheit wird man bei einer Anpassung der tariflichen Arbeitsentgelte an die Insolvenzsituation regelmäßig ausgehen können. Dies gilt auch für den spezielleren ablösenden unternehmensbezogenen Verbandstarifvertrag.

6. These: Die Insolvenz stellt als solche keinen wichtigen Grund für die außerordentliche Kündigung eines Werkstarifvertrages dar. Eine außerordentliche Kündigung kommt jedoch in Betracht, wenn die Insolvenz ibre wesentliche Ursache in von außen kommenden, scblechterdings nicht vorbersehbaren Ereignissen hat.

In Rechtsprechung und Literatur ist an sich anerkannt, daß Tarifverträge aus wichtigem Grund außerordentlich gekündigt werden können ${ }^{11}$. Indessen wird man dem Eintritt der Insolvenz für sich allein das Gewicht eines Grundes zur außerordentlichen Kündigung des Tarifvertrages nicht zubilligen können. Der auf Zeit abgeschlossene Tarifvertrag soll die Arbeitsbedingungen für den vorgesehenen Zeitraum gerade unabhängig von der Veränderung der wirtschaftlichen Verhältnisse auf der einen oder anderen Seite festlegen. Das hier liegende Risiko wird von beiden Tarifpartnern bewußt übernommen und kann nicht ohne Zustimmung auf den anderen Vertragsteil abgewälzt werden ${ }^{12}$. Es liegt nicht anders als bei der Anwendung der Grundsätze über den Wegfall der Geschäftsgrundlage. Auch diese können dort nicht eingreifen, wo die Umstände, die zur Erschwerung der Erfüllung der Leistungspflicht oder zur Erhöhung oder Verminderung des Wertes einer Leistung führen, in den Risikobereich des betroffenen Vertragsteiles fallen ${ }^{13}$.

Anders liegt es aber, wenn die Insolvenz in von außen kommenden, schlechterdings nicht vorhersehbaren Ereignissen - Wiedemann/Stumpf nennen das

10 BAG vom 14.3.1961, AP Nr. $78 \mathrm{zu} \ 242$ BGB Ruhegehalt $=$ DB 1961, 881 für die Bezugnahme auf eine betriebliche Ruhegeldregelung.

11 Vgl. Wiedemann/Stumpf, aaO (Fn. 3), \$4 TVG Anm. 22ff; DÄUbler/Hege, aaO (Fn. 6), Anm. $657 \mathrm{ff}$ jeweils m. Nachw.

12 Vgl. Wiedemann/STumpF, aaO (Fn. 3), \4 TVG Anm. 28.

13 Siehe etwa BGH vom 6.7.1964, LM Nr. $47 \mathrm{zu} \ 242$ (B6) BGB $=$ BB 1964, 944; Roth, MünchKomm. z. BGB, $\$ 242$ Anm. 487 f; Solrgel/SiEBERT/Knopp, Komm. z. BGB, 10. Aufl., $\$ 242$ Anm. 369; ausführlich UlmeR, AcP 174 (1974), $167 \mathrm{ff}, 181 \mathrm{ff}$. 
Verbot der Produktion, die Liefersperre von Rohstoffen oder die Ölkrise - ihre wesentliche Ursache hat. Derartige unvorhersehbare Ereignisse können nicht mehr als in den Risikobereich der Tarifvertragsparteien und ihrer Mitglieder fallend angesehen werden und müssen deshalb dort zur außerordentlichen Kündigung des Tarifvertrags berechtigen, wo dessen weitere Erfüllung, weil zur Insolvenz eines Unternehmens führend, unzumutbar wird ${ }^{14}$.

7. These: Der Austritt des insolventen Unternebmens aus dem Arbeitgeberverband beendet die Bindung an die laufenden Tarifverträge nicht ( 3 Abs. 3 TVG). Die Insolvenz als solche ermöglicht auch keine außerordentliche Kündigung der Tarifbindung. Doch kommt auch insoweit eine außerordentliche Kündigung in Betracht, wenn die Insolvenz ibre wesentliche Ursache in von außen kommenden, schlechterdings nicht vorbersebbaren Ereignissen hat.

Durch den Austritt aus dem Arbeitgeberverband kann sich ein insolvent gewordenes Unternehmen nach der Regelung des $\$ 3$ Abs. 3 TVG nur von der Bindung an künftige Tarifverträge, nicht von der Bindung an die zum Zeitpunkt des Austritts bestehenden Tarifverträge lösen. Allerdings wird man einem aus dem Arbeitgeberverband ausgetretenen Mitglied die Möglichkeit einräumen müssen, sich bei Vorhandensein eines wichtigen Grundes durch außerordentliche Kündigung von der Tarifbindung zu lösen. Das ist ein notwendiges Äquivalent dafür, daß das ausgetretene Mitglied keinen Einfluß mehr auf die Entscheidung über Aufrechterhalten oder Kündigen des Tarifvertrages durch den Arbeitgeberverband hat. Indes stellt auch insoweit die Insolvenz als solche noch keinen wichtigen Grund für die außerordentliche Kündigung dar. Vielmehr ist ein solcher Grund auch hier nur bei von außen kommenden, schlechterdings unvorhersehbaren Ereignissen gegeben, sofern diese wesentlich zur Insolvenz des betreffenden Unternehmens geführt haben.

8. These: Durch Betriebsvereinbarung oder arbeitsvertragliche Abrede können tariflich festgelegte Entgelte nur angepaßt werden, wenn dies der bisherige oder ein neuer Tarifvertrag durch eine Öffnungsklausel gestatten.

Weder die arbeitsvertragliche Abrede noch die Betriebsvereinbarung sind in der Lage, tariflich festgelegte Entgelte zum Nachteil des Arbeitnehmers zu verändern. Der Tarifvertrag setzt Rechtsnormen, die gegenüber dem Arbeitsvertrag und, wie die Tarifsperren der $\$ \$ 87$ Abs. 1 und 77 Abs. 3 BetrVG zeigen, auch gegenüber der Betriebsvereinbarung zwingend sind. Eine Abweichung zum

14 Zum Eingreifen der Grundsätze über den Wegfall der Geschäftsgrundlage im Falle nicht vorhersehbarer Änderungen der bei Abschluß des Vertrags vorausgesetzten Verhältnisse vgl. vor allem ULMER, AcP 174 (1974), $185 \mathrm{ff}$. 
Nachteil der Arbeitnehmer ist deshalb nur möglich, wenn der Tarifvertrag selbst eine Abweichung gestattet ( $\$ 4$ Abs. 3 TVG) oder wenn die Tarifvertragsparteien ihre Zustimmung zu einem vergleichsweise erfolgenden Verzicht auf die tariflichen Rechte gehen ( $\$ 4$ Abs. 4 Satz 1 TVG).

Der von Vollmer gemachte Vorschlag, aus $\$ 111$ BetrVG eine betriebliche „Annex-Kompetenz" zur Kürzung tariflich festgelegter Leistungen für den Fall abzuleiten, daß die Existenz des Unternehmens oder der überwiegende Teil der Arbeitsplätze gefährdet ist und den Arbeitnehmern als Ausgleich für die Kürzung eine angemessene Bestandsgarantie für die Arbeitsplätze gegeben wird ${ }^{15}$, scheitert an dem klaren Wortlaut des BetrVG. Dieses hat in den $\$ \int 87 \mathrm{Abs} .1$ und 77 Abs. 3 eine umfassende Tarifsperre festgelegt und in $\$ 112 \mathrm{Abs} .1$ Satz 4 eine Ausnahme nur für den Sozialplan, also für eine Regelung zugunsten der Arbeitnehmer vorgesehen. Davon abgesehen ist die Verwirklichung des Vorschlags rechtspolitisch auch gar nicht wünschenswert, worauf unten bei These 24 noch einzugehen sein wird.

9. These: Eine Anpassung von tariflich festgelegten Arbeitsentgelten für ein insolventes Unternebmen durch Bundesgesetz ist in Ausnabmefällen möglich.

Das Grundrecht der Koalitionsfreiheit (Art. 9 Abs. 3 GG) garantiert Koalitionen die Tarifautonomie mit. Das bedeutet nach der bekannten Entscheidung des Bundesverfassungsgerichts vom 18.11.1954 $4^{16}$ einmal, daß der Staat den Koalitionen „den Kernbereich eines Tarifvertragssystems überhaupt zur Verfügung stellen muß". Und es bedeutet, da die Tarifautonomie als Ausdruck der Koalitionsfreiheit nicht auf Beschränkung, sondern auf möglichst weitgehende Entfaltung ihrer Funktion angelegt ist, weiter, daß der Staat Eingriffe nur vornehmen darf, wenn Erfordernisse des Gemeinwohls dazu nötigen (Grundsatz der Erforderlichkeit) und die Intensität des Eingriffs nicht außer Verhältnis zur Dringlichkeit des zu wahrenden öffentlichen Interesses steht (Grundsatz der Verhältnismäßigkeit $)^{17}$. Dies steht im Regelfall einer staatlichen Festsetzung der Arbeitsbedingungen im Wege. Es schließt sie aber keineswegs völlig aus. Wo es um sachliche Teilgebiete der Arbeitsbedingungen von besonders hohem Allgemeininteresse geht und wo es gilt, zeitlich begrenzt einer besonderen Notstandssituation $\mathrm{zu}$ steuern, ist auch eine staatliche Festsetzung der Arbeitsbedingungen möglich $^{18}$. Daraus folgt, daß jedenfalls dort, wo es um die Insolvenz eines auch für die Allgemeinheit bedeutenden Großunternehmens geht, eine gesetzliche

15 VOLLMER, DB 1982, $1670 \mathrm{ff}$.

16 BVerfGE 4, $107 \mathrm{ff}=\mathrm{AP}$ Nr. 1 zu Art. 9 GG.

17 In diesem Sinne die Formulierung von WERNER WeBER, Koalitionsfreiheit und Tarifautonomie als Verfassungsproblem, 1965, S.34 ff.

18 Vgl. Werner Weber, aaO (Fn. 17); siehe auch Löwisch, RdA 1969, 129, 130. 
Anpassung der Arbeitsentgelte für einen begrenzten Zeitraum möglich ist, wenn dies zur Sanierung des Unternehmens notwendig erscheint und eine Anpassung auf anderem Wege ausscheidet.

\section{Die Anpassung in Betriebsvereinbarungen festgelegter Entgelte}

10. These: In Betriebsvereinbarungen festgelegte Entgelte und sonstige Arbeitgeberleistungen können durch eine neue Betriebsvereinbarung abgelöst werden.

Nicht anders als tarifvertraglich festgelegte Leistungen durch einen neuen Tarifvertrag können in einer Betriebsvereinbarung festgelegte Leistungen, also insbes. die betrieblichen Sozialleistungen wie Ruhegelder, Gratifikationen und Urlaubsgelder, aber auch in Betriebsvereinbarungen festgelegte Entgelte, etwa Zulagen oder die Gehälter für außertarifliche Angestellte, durch eine neue Betriebsvereinbarung auch zum Nachteil der Arbeitnehmer für die Zukunft geändert werden ${ }^{19}$. Dies folgt wie beim Tarifvertrag aus dem Rechtsnormcharakter, der auch der Betriebsvereinbarung zukommt.

11. These: Eine solche ablösende Betriebsvereinbarung kann auch in einem Sozialplan entbalten sein, ist aber, soweit es nicht um Gegenstände des $\int 87$ Abs. 1 BetrVG geht, nicht wie dieser erzwingbar.

Bestimmungen, die in einer Betriebsvereinbarung festgelegte Arbeitsentgelte und sonstige Arbeitsbedingungen anpassen, können auch im Rahmen eines Sozialplans getroffen werden. Freilich nehmen sie nicht an der Erzwingbarkeit des Sozialplans nach $₫ 112$ Abs. 4 BetrVG teil. Denn der erzwingbare Sozialplan hat, wie sich aus $₫ 112$ Abs. 1 Satz 2 BetrVG ergibt, nur die Einigung über den Ausgleich oder die Milderung der wirtschaftlichen Nachteile zum Gegenstand, die den Arbeitnehmern infolge einer Betriebsänderung entstehen. Hier geht es aber ganz im Gegenteil um die Zufügung von Nachteilen an die Arbeitnehmer, und zwar an solche, die gar keine Ausgleichsleistungen erhalten, so daß man von vorneherein auch nicht mit dem Gedanken einer Annexkompetenz zu einer erzwingbaren Regelung operieren kann.

Erzwingbar sind deshalb derartige Anpassungsbestimmungen nur, soweit Gegenstände betroffen sind, die der Mitbestimmung nach $₫ 87$ Abs. 1 BetrVG unterliegen. Soweit es um den Bereich der freiwilligen Mitbestimmung nach $₫ 88$ BetrVG geht - und das ist regelmäßig der Fall, soweit eine Anpassung der

19 BAG (Großer Senat) vom 16.3.1956, AP Nr.1 zu $₫ 57$ BetrVG unter I2; zuletzt KREUTZ, Grenzen der Betriebsautonomie, 1979, S. 247; siehe auch LöwISCH, DB 1983, 1709. 
Entgelte und sonstigen Arbeitgeberleistungen erfolgen soll - kann eine Änderung auch nur durch freiwillige Betriebsvereinbarung i.S. des $\$ 88$ BetrVG getroffen werden. Kommt eine solche nicht zustande, bleibt nur das Recht, die Betriebsvereinbarung gem. $\ 77$ Abs. 5 BetrVG mit einer Frist von 3 Monaten zu kündigen - sofern dieses Recht nicht bei Abschluß der Betriebsvereinbarung ausgeschlossen oder eingeschränkt ist. Eine außerordentliche Kündigung kommt aus den gleichen Gründen wie die außerordentliche Kündigung des Tarifvertrages regelmäßig nicht in Betracht ${ }^{20}$.

\section{These: Die ablösende Betriebsvereinbarung unterliegt einer abstrakten und konkreten Billigkeitskontrolle durch die Arbeitsgerichte.}

Wie das BAG in zwei Entscheidungen vom 8.12.198121 wieder bestätigt hat, müssen Betriebsvereinbarungen, die auf Betriebsvereinbarungen oder auf vertraglichen Einheitsregelungen beruhende kollektive betriebliche Regelungen zum Nachteil der Arbeitnehmer abändern, die Grundsätze von Recht und Billigkeit, insbesondere den Vertrauensschutzgedanken beachten. Dies verdient, wie ich an anderer Stelle näher dargetan habe ${ }^{22}$, Zustimmung. Dabei ist dem BAG auch darin beizupflichten, daß Rechtspositionen, die der Arbeitnehmer durch seine Arbeitsleistung bereits erdient hat, etwa bereits entstandene Lohnansprüche, die durch Arbeitsleistung bereits erdienten Ruhegeldanwartschaften oder der grundsätzlich auch den Vertragsinhalt sichernde Status der Unkündbarkeit, nur unter erschwerten Voraussetzungen entzogen werden dürfen. $\mathrm{Zu}$ diesen gehört allerdings gerade die wirtschaftliche Notlage des Arbeitgebers, die das Unternehmen und damit auch die in ihm vorhandenen Arbeitsplätze gefährdet. Denn daß das Unternehmen und die Arbeitsplätze und damit die Quelle von Arbeitgeberleistungen erhalten bleiben, setzen Arbeitgeber wie Arbeitnehmer

20 Etwas anderes gilt insoweit für sog. „vorsorgliche“ Sozialpläne, die im voraus festlegen, welche Leistungen Arbeitnehmer erhalten, die bei einer künftigen Betriebsänderung Nachteile erleiden. Tritt hier der Fall der Insolvenz ein, muß ein Konkurs- oder Vergleichsverwalter oder ein künftiger Insolvenzverwalter diesen vorsorglichen Sozialplan aus wichtigem Grund kündigen können, soweit sonst die vom BAG geforderte abgewogene Berücksichtigung der Interessen auch der anderen Gläubiger unmöglich gemacht wäre. Vgl. BAG (Großer Senat) vom 13.12.1978, AP Nr. 6 zu $\$ 112$ BetrVG 72 = BB 1979, 267; DieTz/Richardi, Komm. z. BetrVG, 6. Aufl., 1982, $\$ 112$ Anm. 107; Galperin/Löwisch, Komm. z. BetrVG, 6. Aufl., 1982, \$112 Anm. 43 a. Wird ein Sozialplan direkt im Hinblick auf einen bevorstehenden Konkurs aufgestellt, sind außerdem die Konkursanfechtungsbestimmungen der $\$ \$ 30,31 \mathrm{KO}$ anwendbar. Vgl. LAG Hamm vom 20.1.1982, DB 1982, 1119; WrLLEMSEN, ZIP 1982, 649 ff; GALPERIN/Löwrsch, aaO, $\$ 112$ BetrVG Anm. 74.

21 BAG AP Nr. 1 zu $\int 1$ BetrAVG - Ablösung = BB 1981, $2139=$ DB 1982, 46; BAG AP - Nr. 1 zu $\ 87$ BetrVG 1972 Unterstützungskassen = DB 1982, 50.

22 LöwISCH, DB 1983, 1709, $1711 \mathrm{ff}$. 
als selbstverständlich voraus, wenn sie solche Arbeitgeberleistungen festsetzen. Deshalb ist es unter dem Gesichtspunkt des Wegfalls der Geschäftsgrundlage möglich, diese Leistungen so anzupassen, daß die Gefahr für das Unternehmen und die in ihm vorhandenen Arbeitsplätze beseitigt wird.

Von vornherein weiter ist die Befugnis zur Schaffung ablösender Betriebsvereinbarungen in dem Bereich, in dem es um blo $\beta$ in Aussicht stebende künftige Ansprüche des Arbeitnehmers geht. Auch hier muß aber mit dem BAG eine eingeschränkte Billigkeitskontrolle anerkannt werden, wobei auch der Unterscheidung zwischen einer „abstrakten “ Billigkeitskontrolle, welche die abändernde Betriebsvereinbarung insgesamt betrachtet, und einer „konkreten “ Billigkeitskontrolle, die in einer zweiten Stufe überprüft, ob eine insgesamt billigenswerte Neuregelung für den Einzelfall korrigiert werden muß, weil sie dort zu unbilligen Härten führt, beizupflichten ist. Praktisch bedeutet das für die Insolvenzsituation einerseits, daß das durch den Sanierungszweck ausgelöste Bedürfnis zur Anpassung der Arbeitgeberleistungen abzuwägen ist gegen das Vertrauen des Arbeitnehmers auf den in Aussicht stehenden Erwerb künftiger Ansprüche. Dieses Vertrauen fällt umsomehr ins Gewicht, je näher der Zeitpunkt des Anspruchserwerbs schon gerückt ist und der Arbeitnehmer sich dementsprechend, etwa in seiner Versorgungsplanung, eingerichtet hat, und je größer die Einbuße ist, die ihm durch die Änderung zugemutet wird. Andererseits wird nachgeprüft werden müssen, ob die unterschiedlichen in Betriebsvereinbarungen und vertraglichen Einheitsregelungen festgelegten Leistungen in einem rechten Verhältnis zueinander angepaßt worden sind. So könnte es möglicherweise unbillig sein, wenn die Anpassung ausschließlich bei den Anwartschaften auf betriebliche Ruhegelder vorgenommen würde, während andere Sozialleistungen und die übertariflichen Entgelte, etwa Zulagen, gänzlich unberührt blieben.

Soweit es um die konkrete Billigkeitskontrolle geht, führt im übrigen die Feststellung eines Verstoßes gegen die Grundsätze der Billigkeit im Einzelfall nicht zur Unwirksamkeit der ablösenden Betriebsvereinbarung insgesamt, sondern nur dazu, daß die Betriebsvereinbarung so korrigiert wird, daß eine unbillige Benachteiligung einzelner Belegschaftsmitglieder oder einer Gruppe von ihnen vermieden wird. Die daraus möglicherweise resultierende höhere Belastung des Unternehmens muß in Kauf genommen werden ${ }^{23}$.

13. These: In Betriebsvereinbarungen festgelegte Entgelte und sonstige Leistungen können auch durcb Tarifvertrag angepaßt werden. Ein derartiger Tarifvertrag muß den Vertrauensschutzgedanken berücksichtigen.

$23 \mathrm{Vgl}$. für den parallelen Fall der Billigkeitskontrolle eines Sozialplans BAG vom 17.2. 1981, AP Nr. 11 zu $\$ 112$ BetrVG $1972=$ BB 1981, $1092=$ DB 1981, 1414. 
Angesichts des Vorrangs, der dem Tarifvertrag gemäß $\$ \$ 87$ Abs. 1 und 77 Abs. 3 BetrVG gegenüber der Betriebsvereinbarung zukommt, kann eine Anpassung von in Betriebsvereinbarungen festgelegten Entgelten und sonstigen Arbeitgeberleistungen auch durch Tarifvertrag, insbesondere einen Werkstarifvertrag erfolgen ${ }^{24}$. Ein derartiger ablösender Tarifvertrag unterliegt anders als die ablösende Betriebsvereinbarung keiner gerichtlichen Billigkeitskontrolle ${ }^{25}$. Jedoch muß auch ein solcher Tarifvertrag den Gedanken des Vertrauensschutzes beachten, insbesondere soweit es um den Eingriff in bereits erdiente Ansprüche und Anwartschaften geht. Freilich wird dieser Vertrauensschutz auch hier gegenüber der Notwendigkeit, einer wirtschaftlichen Notlage des Unternehmens Rechnung zu tragen, regelmäßig zurücktreten. Ein die Betriebsvereinbarung ablösender Tarifvertrag ist im übrigen auch an den allgemeinen Gleichheitssatz des Art. 3 Abs. 1 GG gebunden ${ }^{26}$. Willkürliche Ungleichbehandlungen bei der Anpassung müssen also vermieden werden.

14.These: In Ausnabmefällen ist auch eine Anpassung durch Bundesgesetz möglich.

Nicht anders wie die tariflich festgelegten Arbeitsentgelte können auch die in Betriebsvereinbarungen festgelegten Arbeitsentgelte im Ausnahmefall durch Bundesgesetz angepaßt werden. Das Grundrecht des Art. 9 Abs. 3 GG, welches nur den Koalitionen, nicht aber Arbeitgeber und Betriebsrat als Partner der Betriebsvereinbarung zusteht, steht hier von vorneherein nicht entgegen.

\section{Die Anpassung einzelvertraglich festgelegter Entgelte}

15. These: Einzelvertraglich festgelegte Entgelte und sonstige Arbeitgeberleistungen können durch eine vereinbarte Änderung des Arbeitsvertrages angepaßt werden. Die Grundsätze einer solchen Anpassung bedürfen, wenn es sich um eine vertragliche Einheitsregelung bandelt und ein Gegenstand des $\int 87$ Abs. 1 BetrVG betroffen ist, der Zustimmung des Betriebsrats.

Der unproblematischste Weg, um einzelvertraglich festgelegte Entgelte und sonstige Arbeitgeberleistungen der Insolvenzsituation anzupassen, ist an sich der der vertraglichen Vereinbarung zwischen Arbeitgeber und Arbeitnehmer.

24 BAG vom 17.4.1959, AP Nr.1 zu $\ 4$ TVG Günstigkeitsprinzip = BB 1959, 704; Galperin/Löwisch, aaO (Fn. 20), \$77 BetrVG Anm. 70; Dietz/Richardi, aaO (Fn. 20), §77 BetrVG Anm. 212; Wiedemann/Stumpf, aaO (Fn.3), \$4 TVG Anm. 296; a. A. ThIELE, Gemeinschaftskomm. z. BetrVG, $\$ 77$ Anm. 62.

25 Vgl. BAG DB 1983, 944.

26 Vgl. BAG DB 1983, 944. 
Erfolgt diese Vereinbarung allerdings in einer vertraglichen Einheitsregelung, so ist, soweit eine mitbestimmungspflichtige Angelegenheit i.S. des $\$ 87$ Abs. 1 BetrVG betroffen ist, zu deren Grundlinien die Zustimmung des Betriebsrats in Form der sog. Regelungsabrede erforderlich ${ }^{27}$.

16. These: Einseitig ist eine Anpassung möglich, soweit sich der Arbeitgeber das Recht zum Widerruf einer Leistung vorbebalten bat. Wo das nicht der Fall ist, ist der Arbeitgeber auf den Weg der Änderungskündigung verwiesen; diese muß die Kündigungsfristen einbalten und kann von den Arbeitsgerichten auf ibre soziale Rechtfertigung kontrolliert werden. Auch bier bedürfen die Grundsätze einer solchen Anpassung der Zustimmung des Betriebsrats, sowie es sich um eine vertragliche Einheitsregelung handelt und ein Gegenstand des $\int 87$ Abs. 1 BetrVG betroffen ist.

Soweit sich der Arbeitgeber das Recht zum Widerruf zugesagter Leistungen vorbehalten hat, kann er durch dessen Ausübung eine von ihm für notwendig gehaltene Änderung der Arbeitsbedingungen herbeiführen. Allerdings muß er sich dabei im Rahmen billigen Ermessens halten. Die Maßstäbe des billigen Ermessens sind dabei nicht notwendig die der sozialen Rechtfertigung i.S. des Kündigungsschutzgesetzes. Vielmehr kann die Ausübung eines vorbehaltenen Widerrufs schon beim Vorliegen „betrieblicher Gründe" und nicht erst beim Vorliegen "dringender" betrieblicher Gründe zugelassen $\operatorname{sein}^{28}$. Jedenfalls stellt die Notwendigkeit, einer Insolvenzsituation Rechnung zu tragen, einen Grund dar, aus dem ein solcher Widerruf billigerweise erfolgen kann ${ }^{29}$.

Im übrigen bleibt dem Arbeitgeber gegenüber den Arbeitnehmern, die einer Anpassung ihrer einzelvertraglich festgelegten Entgelte und sonstigen Arbeitgeberleistungen nicht zustimmen, wie auch sonst der Weg der Änderungskündigung. Diese muß die Kündigungsfristen einhalten, wobei an die Stelle vertraglich vereinbarter Kündigungsfristen die gesetzlichen Kündigungsfristen treten, wenn man für den Sanierungsfall eine dem $\$ 22 \mathrm{KO}$ entsprechende Regelung triff ${ }^{30}$. Derartige Änderungskündigungen können mit der Kündigungsschutzklage nach $\$ \int 2,8 \mathrm{KSchG}$ angegriffen werden und sind in diesem Falle vom Arbeitsgericht auf ihre soziale Rechtfertigung zu überprüfen. Diese wird im wesentlichen unter den gleichen Voraussetzungen zu bejahen sein, unter denen eine entsprechende ablösende Betriebsvereinbarung als billig anzusehen wäre. Insbesondere reicht es

27 So für den Fall der Ausübung eines Widerrufsvorbehalts BAG vom 17.12. 1980, AP Nr. 4 zu $\ 87$ BetrVG 1972 Lohngestaltung = BB 1981, $789=$ DB 1981, 1045.

28 BAG vom 7.10.1982, DB 1983, 1368.

29 Zutreffend HANAU in seinem Gutachten für den 54. Deutschen Juristentag, Verhandlungen des 54. DJT, Bd.1, S.E27.

30 Zur Notwendigkeit einer solchen Regelung vgl. LöwISCH, ZIP 1981, 1288, 1292. 
also auch hier aus, wenn nachgewiesen wird, daß die Anpassung notwendig ist, um die Gefährdung des Unternehmens und seiner Arbeitsplätze abzuwenden.

In den Fällen, in denen vertraglich oder tarifvertraglich die ordentliche Kündigung ausgeschlossen ist, wie das für Arbeitnehmer mit längerer Betriebszugehörigkeit nach Erreichen eines bestimmten Lebensalters immer häufiger vorkommt, ist auch eine außerordentliche Änderungskündigung möglich. Da derartige Vereinbarungen aber in der Regel so zu verstehen sind, daß gerade auch die ordentliche Änderungskündigung ausgeschlossen sein soll, um den Arbeitnehmern so ihren einmal erreichten Status ungeschmälert zu erhalten, kann man einen wichtigen Grund für die außerordentliche Änderungskündigung in solchen Fällen nur annehmen, wenn eine Situation gegeben ist, die dem des Wegfalls der Geschäftsgrundlage entspricht. Das wird allerdings dann anzunehmen sein, wenn angesichts der Insolvenzsituation die Aufrechterhaltung der bisherigen Entgelte das Unternehmen und damit die Arbeitsplätze gefährdet.

Auch bei einer durch Ausübung eines Widerrufsvorbehalts oder durch Änderungskündigung vorgenommenen Anpassung gilt, daß für deren Grundsätze die Zustimmung des Betriebsrats in Form der Regelungsabrede vorliegen muß, soweit eine vertragliche Einheitsregelung getroffen werden soll und der Bereich der mitbestimmungspflichtigen Angelegenheiten i.S. des $₫ 87$ Abs. 1 BetrVG betroffen ist ${ }^{31}$.

17. These: Eine Anpassung individuell mit dem einzelnen Arbeitnebmer ausgehandelter Entgelte und sonstiger Arbeitgeberleistungen durch Tarifvertrag oder Betriebsvereinbarung ist wegen des Günstigkeitsprinzips nicht möglich.

Individuell mit dem einzelnen Arbeitnehmer ausgehandelte Arbeitsbedingungen können weder durch Tarifvertrag noch durch Betriebsvereinbarung angepaßt werden. Dem steht das für Tarifverträge in $\$ 4$ Abs. 3 TVG ausdrücklich niedergelegte, aber auch für Betriebsvereinbarungen hinsichtlich dort festgelegter materieller Arbeitsbedingungen geltende ${ }^{32}$ Günstigkeitsprinzip entgegen.

18. These: In vertraglichen Einbeitsregelungen festgelegte Entgelte und sonstige Arbeitgeberleistungen können durch der Billigkeitskontrolle unterliegende freiwillige Betriebsvereinbarung angepaßt werden. Die Tarifüblichkeitssperre des \} 7 7 \text { Abs. } 3 \text { BetrVG gilt dabei nicht. }

Mit der bisherigen Rechtsprechung des $\mathrm{BAG}^{33}$ ist davon auszugehen, daß $\$ \$ 87$ und 88 BetrVG Arbeitgeber und Betriebsrat mit der Kompetenz zur

31 Vgl. Fn. 27.

32 Vgl. Galperin/Löwisch, aaO (Fn. 20), $₫ 77$ BetrVG Anm. $94 \mathrm{ff}$.

33 Vgl. insbesondere BAG v. 30.1.1970, AP Nr. $142 \mathrm{zu} \$ 242 \mathrm{BGB}-$ Ruhegehalt $=\mathrm{DB}$ $1970,1393=\mathrm{BB} 1970,1097 ; \mathrm{BAG}$ v. 8.12.1981, AP Nr.1 zu $\$ 1$ BetrAVG Ablösung; a. A. BAG (6.Senat) v. 12.8.1982, BB 1982, 2298. 
Regelung sozialer Angelegenheiten grundsätzlich auch die Befugnis zuweisen, bestehende arbeitsvertragliche Einheitsregelungen einer solchen sozialen Angelegenheit, also inhaltlich gleichlautende, insbesondere auf eine Gesamtzusage zurückgehende, Arbeitsbedingungen auch zu Ungunsten der Arbeitnehmer abzuändern. Dem Betriebsrat die Kompetenz zum Abschluß solcher verschlechternder Betriebsvereinbarungen mit der Begründung zu verwehren, dies vertrage sich nicht mit seiner Pflicht, als Repräsentant der Belegschaft deren Interesse gegenüber dem Arbeitgeber wahrzunehmen, verkennt die Stellung, die dem Betriebsrat nach dem BetrVG zukommt. Dieses sieht ihn nicht allein als Vertreter der Individualinteressen der einzelnen Arbeitnehmer an. Vielmehr verpflichtet es ihn in $\$ 2 \mathrm{Abs} .1$ zur Berücksichtigung des Wohls „,der Arbeitnehmer und des Betriebes". Dieser Verpflichtung kann er nur gerecht werden, wenn ihm die Befugnis zusteht, im Interesse der Gesamtbelegschaft oder des weiteren Gedeihens des Betriebes auch einmal verschlechternd in die durch vertragliche Einheitsregelung verliehenen Ansprüche bestimmter Arbeitnehmergruppen einzugreifen ${ }^{34}$.

Wo die vertragliche Einheitsregelung allerdings dahin zu verstehen ist, daß die in ihr zugesagten Leistungen der Arbeitnehmer auch gegenüber einer späteren kollektiven Änderung bestandsfest garantiert sein soll, ist die spätere Abänderung durch Betriebsvereinbarung unzulässig, weil sie gegen das Günstigkeitsprinzip verstoßen würde. Von einem solchen Willen des Arbeitgebers, mit der vertraglichen Einheitsregelung „für den Arbeitnehmer einen individuell geprägten Vertrauenstatbestand zu schaffen ${ }^{(35}$ wird man angesichts ihrer erheblichen Bedeutung für die einzelnen Arbeitnehmer etwa bei der arbeitsvertraglich erfolgten Festlegung der regelmäßigen Wochenarbeitszeit sprechen können. Sie kann nur vorübergehend - durch Einführung von Kurzarbeit und Mehrarbeit - nicht aber auf Dauer durch Betriebsvereinbarung geändert werden. In der Regel aber ist die Anpassung von Entgelten und anderen Arbeitgeberleistungen durch ablösende Betriebsvereinbarung möglich.

Eine solche ablösende Betriebsvereinbarung unterliegt nicht anders als die Betriebsvereinbarung, die durch Betriebsvereinbarung festgelegte Entgelte und andere Arbeitgeberleistungen anpaßt, der Billigkeitskontrolle durch die Arbeitsgerichte. Nach richtiger Meinung steht entsprechenden Betriebsvereinbarungen die Tarifsperre des $\$ 77$ Abs. 3 BetrVG auch dort nicht entgegen, wo die in der vertraglichen Einheitsregelung festgelegte Arbeitgeberleistung sich auf einen tariflich geregelten Gegenstand bezieht: Die Sperre des $\$ 77$ Abs. 3 BetrVG hat lediglich den $Z$ weck, den Tarifvertragsparteien das Privileg zur inhaltlichen Ausgestaltung von materiellen Arbeitsbedingungen zu sichern. Dieses Privileg ist nicht berührt, wenn eine Betriebsvereinbarung sich darauf beschränkt, bisher

34 Vgl. näher Löwrsch, DB 1983, $1709 \mathrm{ff}$.

35 RICHARDI, Anm. zu BAG AP Nr. 142 zu $§ 242$ BGB - Ruhegehalt. 
bestehende betriebliche Regelungen lediglich zu beseitigen oder einzuschränken, ohne neue an die Stelle zu setzen ${ }^{36}$.

19. These: Gegenüber leitenden Angestellten bestebt die Möglichkeit, arbeitsvertragliche Einheitsregelungen durch Betriebsvereinbarung anzupassen, nicht.

In bezug auf die leitenden Angestellten fehlt Arbeitgeber und Betriebsrat die Kompetenz zum Erlaß von Betriebsvereinbarungen ${ }^{37}$. Wenn Stump $f^{38}$ unter Hinweis auf das Gleichnis vom guten und schlechten Tropfen meint, die Einbeziehung der leitenden Angestellten in die durch die ablösende Betriebsvereinbarung bewirkte verschlechternde Neuregelung müsse ebenso möglich sein wie ihre vom $\mathrm{BAG}^{39}$ erwogene Einbeziehung in Sozialplanregelungen, so kann das nicht i.S. einer automatischen Geltung des Inhalts der ablösenden Betriebsvereinbarung für die leitenden Angestellten gemeint sein. Arbeitgeber und Betriebsrat haben nach $\ 5$ Abs. 3 BetrVG gegenüber den leitenden Angestellten keine Kompetenz zur Rechtsnormsetzung und eine entsprechende schuldrechtliche Vereinbarung zwischen ihnen wäre nichts anderes als ein Vertrag zu Lasten Dritter. Denkbar ist allenfalls, daß sich der Arbeitgeber gegenüber dem Betriebsrat verpflichtet, eine entsprechende Neuregelung auf arbeitsvertraglichem Wege auch gegenüber den leitenden Angestellten durchzusetzen. Darin läge wohl und das ist der richtige Kern des Gedankens von Stumpf-dann keine Umgebung des $\$ 5$ Abs. 3 BetrVG, wenn die abzulösende Betriebsvereinbarung gleichermaBen für normale Arbeitnehmer und leitende Angestellte gegolten hatte ${ }^{40}$.

20. These: Eine Anpassung in arbeitsvertraglichen Einheitsregelungen festgelegter Entgelte oder sonstigen Arbeitgeberleistungen ist auch durch Tarifvertrag möglich. Ein entsprechender Tarifvertrag kann auch für allgemeinverbindlich erklärt werden.

36 Vgl. ZöllNER, 2. FS Nipperdey, Bd. 2, 1965, S.699, $719 \mathrm{ff}$.

37 v. Hoyningen-HuEne, 2. FS Nipperdey, Bd. 2, 1965, S. $231 \mathrm{f}$.

38 STUMPF, Die ablösende Betriebsvereinbarung, in: Gegenwart und Zukunft der betrieblichen Altersversorgung, 1982, S. 102, 106.

39 BAG vom 31.1.1979, AP Nr. 8 zu $\$ 112$ BetrVG $1972=$ DB 1979, $1039=$ DB 1979, 833.

40 Weitergehend will v. HoYNINGEN-HuENe, 2. FS Nipperdey, S. 231 f, ablösende Sozialpläne „über den Gleichbehandlungsgrundsatz individualrechtlich“ für leitende Angestellte gelten lassen, weil im verbleibenden Restbestand des reduzierten Sozialplans immer noch eine Begünstigung liege. Dem vermag ich nicht zu folgen. Selbst wenn man, was ich nicht für richtig halte (vgl. dazu LöwISCH, FS G. Müller, 1981 S. $301 \mathrm{ff}$ ), die begünstigenden Wirkungen eines Sozialplans qua Gleichbehandlung inividualrechtlich auf leitende Angestellte erstreckt, führt kein Weg daran vorbei, daß die Erstrekkung einer späteren durch Betriebsvereinbarung erfolgenden Verschlechterung des Sozialplans auf leitende Angestellte auf einen Vertrag zu Lasten Dritter hinausläuft. 
Nach der - freilich nicht unumstrittenen - Rechtsprechung des BAG ${ }^{41}$ kann eine arbeitsvertragliche Einheitsregelung auch durch Tarifvertrag zum Nachteil der Arbeitnehmer verändert werden.

Meines Erachtens kann ein solcher Tarifvertrag, also ein entsprechender Werkstarifvertrag oder unternehmensbezogener Verbandstarifvertrag auch für allgemeinverbindlich erklärt werden: Die Voraussetzung des $\ 5$ Abs. 1 Nr.1 TVG (Beschäftigung von nicht weniger als $50 \mathrm{v}$. H. der unter den Geltungsbereich des Tarifvertrags fallenden Arbeitnehmer durch den Arbeitgeber) ist in diesem Falle gegeben ${ }^{42}$. Auch daß die Allgemeinverbindlicherklärung im öffentlichen Interesse geboten ist, wie das $\ 5$ Abs. 1 Nr. 2 TVG fordert, wird bejaht werden können. Das öffentliche Interesse i.S. dieser Vorschrift erfordert weder, daß ein allgemeiner sozialer Notstand vorliegt ${ }^{43}$. Noch muß es stets vom Gedanken der Vermeidung von Lohndrückerei und Schmutzkonkurrenz oder dem der Sicherung angemessener Arbeitsbedingungen für die Nichtorganisierten getragen sein. Vielmehr genügt das Interesse der Volkswirtschaft wie der in dem sanierungsbedürftigen Unternehmen beschäftigten Arbeitnehmer, eine für alle Arbeitnehmer geltende Anpassung der Arbeitsbedingungen zu erreichen, die den Erfolg der Sanierung sicherstellt.

\section{B. Die Anpassung der Arbeitsentgelte de lege ferenda}

\section{Die Anpassung nicht tariflich festgelegter Entgelte}

21.These: Die Möglichkeit einer Anpassung künftiger Arbeitsentgelte durch Bescblüsse von Gläubigermebrbeiten im Rabmen des Sanierungsverfabrens ist abzulebnen.

Die künftigen über- und außertariflichen Entgelte und sonstigen Arbeitgeberleistungen zur Abstimmung aller Gläubiger im Sanierungsverfahren zu stellen, wie das etwa Timm vorschlägt ${ }^{44}$ begegnet erheblichen Bedenken. Man kann die anderen Gläubiger nicht als legitimiert ansehen, künftige Arbeitnehmerforderungen, die ja von den Arbeitnehmern erst erarbeitet werden sollen, zu streichen. Es ist dies etwas grundsätzlich anderes, als bestebende Anteilseignerrechte

41 Vgl. BAG v. 4.2.1960, AP Nr.7 zu \4 TVG-Günstigkeitsprinzip = BB 1960, $663=$ DB 1960, 698. Zuvor schon BAG v. 20.12.1957, AP Nr. 11 zu Art. 44 Truppenvertrag $=\mathrm{BB}$ 1958, 304; vgl. WIEDemann/STumpF, aaO (Fn.3), \$4 TVG Anm. $308 \mathrm{ff} \mathrm{m}$. entspr. Nachw.

42 Vgl. Wiedemann/STUMPf, aaO (Fn. 3), \$5 TVG Anm. 28.

43 Vgl. Wiedemann/StumPF, aaO (Fn. 3), \$5 TVG Anm. 29.

44 TIMM, ZIP 1983, 225 ff, 235. 
oder Gläubigerpositionen einer Herabsetzung durch Mehrheitsbeschluß zuzuführen $^{45}$. Hinzu kommt ein weiteres: Jede Möglichkeit, künftige Entgeltansprüche der Arbeitnehmer auch gegen deren Willen herabzusetzen, stellt für die Unternehmen einen Anreiz dar, sich des Sanierungsverfahrens zu diesem $Z$ weck auch dort zu bedienen, wo dessen Einleitung unausweichlich ist. $\mathrm{Daß}$ in den USA kürzlich eine Fluggesellschaft zum erklärten Zweck der Senkung des Personalaufwandes die Einleitung des Sanierungsverfahrens beantragt und eine weitere mit diesem Schritt gedroht hat ${ }^{46}$, mahnt hier wie übrigens auch bei der Umschreibung der Eröffnungstatbestände ${ }^{47}$ zur Vorsicht. Vertretbar ist allein, dem Betriebsrat als Repräsentanten der Arbeitnehmer eine Befugnis zur Verschlechterung der künftigen Arbeitsbedingungen zuzubilligen, sei es, wie das de lege lata schon weitgehend möglich ist, über die ablösende Betriebsvereinbarung, sei es über eine entsprechende, als Teil des Sanierungsverfahrens vorgesehene Befugnis. Letztere Möglichkeit soll hier indessen nicht weiter verfolgt werden, nachdem die Tendenz sowohl des Juristentages wie offenbar der Insolvenzrechtskommission dahin geht, die Auswirkungen des Sanierungsverfahrens auf die Arbeitsentgelte außerhalb des eigentlichen Sanierungsverfahrens mit den herkömmlichen arbeitsrechtlichen Instrumenten zu regeln ${ }^{48}$.

22. These: Ebenfalls abzulehnen ist eine Änderung des geltenden Kündigungsschutzrechts zum Zwecke der Erleichterung der Anpassung künftiger Arbeitsentgelte bei insolventen Unternebmen.

Auch ein Eingriff in das gesetzliche Kündigungsschutzrecht zur Erleichterung des Sanierungsverfahrens ist abzulehnen. Änderungskündigungen, die zur Durchführung eines im Sanierungsverfahren aufgestellten Reorganisationsplans notwendig sind, werden zwar regelmäßig betriebsbedingt i.S. des $₫ 1 \mathrm{Abs.} 2$ KSchG und damit sozial gerechtfertigt sein. Der Sanierungszweck rechtfertigt aber nicht die Aufgabe der gerichtlichen Kontrolle dieser sozialen Rechtfertigung ${ }^{49}$. Die Gefahr des Mißbrauchs bei einer Freigabe der Änderungskündigung, die im Rahmen eines Sanierungsverfahrens erfolgt, ist unübersehbar. Richtig

45 Ablehnend auch HaNAU in seinem Gutachten für den 54. Deutschen Juristentag, aaO (Fn. 29), S.E 106.

46 Vgl. Der Spiegel, Nr. 42/1983, S. $200 \mathrm{ff}$ und den Bericht in der Bad. Zeitung, Freiburg, 30.9.1983.

$47 \mathrm{Vgl}$. hierzu meinen Diskussionsbeitrag bei den Verhandlungen der Abteilung Sanierung von Unternehmen des 54. Deutschen Juristentages, Sitzungsbericht, S. M 97 f.

$48 \mathrm{Vgl}$. die Beschlüsse der Abteilung Sanierung von Unternehmen des 54. Deutschen Juristentages, Sitzungsbericht, S.M $239 \mathrm{f}$, sowie den Bericht von ARNOLD auf dem Juristentag (Sitzungsbericht, S.M $39 \mathrm{ff}$ ).

49 So auch HanAU, aaO (Fn.29), S. E28; a. A. wohl Uhlenbruck, KTS 1981, $513 \mathrm{ff}$, 557. 
erscheint nur, auch für die Änderungskündigung nach dem Vorbild des $\$ 22 \mathrm{KO}$ anzuordnen, daß sie mit der gesetzlichen Kündigungsfrist auch dort erfolgen kann, wo vertraglich längere Kündigungsfristen vereinbart sind.

23. These: Die bereits de lege lata bestehende Möglichkeit in Betriebsvereinbarungen und vertraglichen Einheitsregelungen festgelegte Arbeitsentgelte durch ablösende Betriebsvereinbarung anzupassen, ist wie folgt auszubauen:

a) Über solche Betriebsvereinbarungen entscheidet auch im sonst nicht mitbestimmungspflichtigen Bereich die Einigungsstelle verbindlich.

b) Durch ablösende Betriebsvereinbarung in Sanierungsfällen angepaßte Arbeitsentgelte können bei Änderung der bei Abschluß zugrundegelegten Verbältnisse durch erzwingbare Betriebsvereinbarungen wieder auf den früberen Stand angehoben werden.

c) Die Tarifsperre des $₫ 77$ Abs. 3 BetrVG gilt für derartige Betriebsvereinbarungen nicht.

d) Die Kompetenz zu einer derartigen ablösenden Betriebsvereinbarung wird auch auf individuell ausgehandelte Entgelte und sonstige Arbeitgeberleistungen erstreckt.

Das Gros der nicht tariflich festgelegten Entgelte und sonstigen Arbeitgeberleistungen unterfällt lediglich der freiwilligen Mitbestimmung nach $\$ 88$ BetrVG. Dies bedeutet praktisch, daß eine Anpassung durch ablösende Betriebsvereinbarung nur einvernehmlich zwischen Arbeitgeber und Betriebsrat möglich ist. Dies paßt nur zu einem Konzept des Sanierungsverfahrens, das insgesamt auf die einvernehmliche Regelung der die Arbeitnehmer betreffenden Fragen zwischen Betriebsrat einerseits und den anderen Gläubigern sowie dem Verwalter vertraut, wie das den von mir gemachten Vorschlägen entspricht ${ }^{50}$. Legt man, wie die Konzepte der Insolvenzrechtskommission und auch des Deutschen Juristentages $^{51}$, die Regelung der die Arbeitnehmer betreffenden Fragen im Sanierungsverfahren so an, daß in Streitfällen eine verbindliche Entscheidung durch eine Einigungsstelle zu erfolgen hat, so bleibt man auf halbem Wege stehen, wenn man die Anpassung der nicht tariflich festgelegten Arbeitsentgelte nur der einvernehmlichen Regelung zwischen Arbeitgeber und Betriebsrat öffnet. Praktisch schließt man damit, abgesehen von der - dispositiven - Kündigungsmöglichkeit des $\$ 77 \mathrm{Abs.} 5$ BetrVG, die Änderung in Betriebsvereinbarungen festgelegter Leistungen gegen den Willen des Betriebsrats aus und verweist das Unternehmen bezüglich der einzelvertraglich festgelegten Leistungen auf den schwer praktikablen und kostenaufwendigen Weg der Änderungskündigung.

$50 \mathrm{Vgl}$. Löwrsch, ZIP 1981, 1289 f.

51 Vgl. die Nachweise in Fn. 48. 
Dies drängt dazu, im Zusammenhang mit der Regelung des Sanierungsverfahrens den vom Deutschen Juristentag gemachten Vorschlag, die Möglichkeit der Herabsetzung über- und außertariflicher Entgelte durch Betriebsvereinbarung vorzusehen $^{52}$, dahin zu konkretisieren, daß dabei auch die Möglichkeit der verbindlichen Entscheidung der Einigungsstelle eingeschlossen ist. Soweit eine Betriebsvereinbarung abzulösen ist, könnte dabei dann auch auf deren vorgängige - ordentliche oder außerordentliche - Kündigung verzichtet werden.

Entschließt man sich zu diesem Schritt, muß es dann auch möglich sein, bei Veränderung der zugrundegelegten Umstände durch eine abändernde erzwingbare Betriebsvereinbarung, die angepaßten Entgelte und anderen Arbeitgeberleistungen wieder auf den früheren Stand anzuheben. Die Notwendigkeit dazu ist insbesondere für den Fall unabweisbar, daß, nachdem über die ablösende Betriebsvereinbarung entschieden ist, im Reorganisationsplan nur wesentlich geringere Opfer der anderen Gläubiger und der Anteilseigner vorgesehen werden, als sie Betriebsrat und Arbeitgeber oder Einigungssstelle bei der Festlegung der Opfer der Arbeitnehmerseite zugrundegelegt haben. Aber auch bei einer unvorhergesehenen Verbesserung der wirtschaftlichen Verhältnisse des sanierungsbedürftigen Unternehmens muß eine solche Wiederanhebung der Arbeitsbedingungen möglich sein. So wie ein Sozialplan durch eine spätere Betriebsvereinbarung in den Grenzen von Recht und Billigkeit auch zu Ungunsten der Arbeitnehmer abgeändert werden $\mathrm{kann}^{53}$, muß auch die Änderung einer im Zusammenhang mit der Sanierung erfolgten verschlechternden Betriebsvereinbarung zugunsten der Arbeitnehmer möglich sein.

Klargestellt werden sollte, daß die Tarifsperre des $\$ 77$ Abs. 3 BetrVG für derartige Betriebsvereinbarungen nicht gilt. Dies entspricht zwar für die ablösenden Betriebsvereinbarungen schon jetzt der h. M..$^{54}$, ist aber für Betriebsvereinbarungen, die verschlechterte Arbeitsbedingungen wieder auf den alten Stand anheben, nicht selbstverständlich.

Die Kompetenz zum Erlaß einer erzwingbaren ablösenden Betriebsvereinbarung sollte auch auf individuell ausgehandelte Entgelte und sonstige Arbeitsbedingungen erstreckt werden. Auch die Inhaber solcher individuell ausgehandelter Ansprüche können von einem Sanierungsbeitrag nicht verschont bleiben. Das Unternehmen insoweit auf den umständlichen Weg der Änderungskündigung zu verweisen, entspricht nicht der Notwendigkeit, im Rahmen des Sanierungsverfahrens schnelle Regelungen herbeizuführen. Ein materieller Rechtsnachteil ist mit einer solchen Erstreckung der Kompetenz zum Erlaß ablösender Betriebsvereinbarungen nicht verbunden, weil diese der arbeitsgerichtlichen Billigkeits-

52 Vgl. Beschlüsse des 54. Deutschen Juristentages, aaO (Fn. 48), unter 15.

53 Vgl. BAG vom 24.3.1981, AP Nr. 12 zu $₫ 112$ BetrVG $1972=$ DB 1981, 2178.

54 Vgl. die Nachweise in Fn. 36. 
kontrolle unterliegen, welche der arbeitsgerichtlichen Kontrolle der Änderungskündigung auf soziale Rechtfertigung gleichwertig ist.

Dagegen sehe ich keine Möglichkeit, die Kompetenz zur ablösenden Betriebsvereinbarung auch gegenüber leitenden Angestellten zu begründen: Ihnen gegenüber fehlt dem Betriebsrat die Legitimation, die er gegenüber den anderen Arbeitnehmern des Betriebs durch seine Wahl hat. Insoweit bleibt nur die oben schon dargelegte Möglichkeit, daß sich der Arbeitgeber gegenüber dem Betriebsrat verpflichtet, eine entsprechende Neuregelung auf arbeitsvertraglichem Wege auch gegenüber den leitenden Angestellten durchzusetzen.

\section{Die Anpassung tariflich festgelegter Arbeitsentgelte}

24. These: Ein Recht des insolventen Unternehmens, sich wegen der Insolvenz aus der Tarifbindung zu lösen, ist abzulebnen.

Wenn auch nicht zu verkennen ist, daß es in manchen Fällen, nämlich dort wo auch die Zusatzleistungen zum Arbeitsentgelt weitgehend tariflich geregelt sind, ein Bedürfnis auf Anpassung auch der tariflichen Entgelte gegen den Willen der tarifschließenden Gewerkschaft geben kann, läßt sich ein Recht des insolventen Unternehmens, sich entgegen $\$ 3$ Abs. 3 TVG aus der Tarifbindung zu lösen, nicht befürworten. $\mathrm{Ob}$ man nun ein automatisches Erlöschen der Tarifbindung im Sanierungsfall anordnen ${ }^{55}$ oder dem Unternehmen ein Recht zur Aufhebung der Tarifbindung einräumen würde: Beides liefe darauf hinaus, daß das mit dem Abschluß des Tarifvertrages übernommene Risiko der Erfüllbarkeit der vereinbarten Arbeitsbedingungen für die vereinbarte Zeit dem Unternehmen einfach abgenommen würde. Dafür besteht kein zureichender Grund. Dem Unternehmer muß die Möglichkeit der Kündigung der Tarifbindung bei Vorliegen eines wichtigen Grundes nach Maßgabe des eben bei These 7 Gesagten genügen. Dazu begegnet es Bedenken, auf diese Weise die Tarifvertragsparteien aus ihrer Verantwortung für die Arbeitsbedingungen der Arbeitnehmer auch im sanierungsbedürftigen Unternehmen einfach hinauszudrängen. Tarifautonomie bedeutet Verantwortung der Tarifvertragsparteien für die Arbeitsbedingungen nicht nur in Schönwetter-, sondern auch in Schlechtwetterlagen.

25. These: Abzulebnen ist auch der Vorscblag, tariflich festgelegte Entgelte im Insolvenzfalle durch erzwingbare Betriebsvereinbarungen anzupassen.

Den gleichen Bedenken begegnet der Vorschlag, tariflich festgelegte Arbeitsbedingungen im Insolvenzfalle durch erzwingbare Betriebsvereinbarung anpas- 
sen $\mathrm{zu}$ lassen ${ }^{56}$. Eine solche Regelung würde geradezu eine Umkehrung des in den $\int \$ 77$ Abs. 3 und 87 Abs. 1 BetrVG zum Ausdruck gekommenen Prinzips bedeuten, daß der Regelung der Arbeitsbedingungen durch die freigebildete Koalition der Vorrang gebührt vor der Regelung zwischen Arbeitgeber und Betriebsrat. Für eine solche Umkehrung ist kein Anlaß, jedenfalls so lange nicht, wie es keine umfassenden praktischen Erfahrungen des Inhalts gibt, daß sich die Tarifvertragsparteien, insbesondere die Gewerkschaften, der Aufgabe der Anpassung tariflicher Entgelte an eine Insolvenzsituation entziehen.

$\mathrm{Zu}$ bedenken ist insoweit auch, daß es eine nicht unerhebliche Schwächung der Gewerkschaften bedeuten müßte, wenn ihnen gerade in der Insolvenzsituation die Verantwortung für die Arbeitsbedingungen ihrer Mitglieder genommen würde: Diese würden sich vielfach sagen, daß sie gerade in solchen Situationen auf die Gewerkschaft angewiesen sind und die Mitgliedschaft für sie wenig Wert hat, wenn die Gewerkschaften dann für sie gar nicht tätig werden können. Hier scheint mir durchaus auch ein Verstoß gegen Art. 9 Abs. 3 GG nahezuliegen.

26. These: Vorerst abzulehnen ist auch die Schaffung einer Möglichkeit, tariflich festgelegte Arbeitsbedingungen in einem Zwangsverfahren - etwa nach dem Vorbild des Verfabrens zur Festsetzung von Mindestarbeitsbedingungen - anzupassen.

Eine Möglichkeit, tariflich festgelegte Arbeitsbedingungen gegen den Willen einer Tarifvertragspartei an die Insolvenzsituation anzupassen, besteht de lege lata bis zum Ablauf des Tarifvertrages nicht, soweit nicht ausnahmsweise ein Recht zur außerordentlichen Kündigung des Tarifvertrages gegeben ist. So wie hinsichtlich des Zustandekommens einer ablösenden Betriebsvereinbarung könnte man deshalb auch hinsichtlich des Zustandekommens eines ablösenden Tarifvertrages an die Einführung einer Möglichkeit der verbindlichen Entscheidung in einem $Z$ wangsverfahren denken. Etwa käme in Betracht, eine verbindlich entścheidende Instanz zu schaffen, die ähnlich zusammengesetzt ist wie die Fachausschüsse, die nach dem Gesetz über die Festsetzung von Mindestarbeitsbedingungen über deren Festsetzung entscheiden. Indessen besteht für das Beschreiten eines solchen Weges so lange kein Anlaß, wie nicht festgestellt werden kann, daß sich die Tarifvertragsparteien ihrer Verantwortung für eine einvernehmliche Anpassung auch tariflicher Entgelte entziehen.

56 So TimM, ZIP 1983, 225, 236, sowie KILGER, ZIP 1982, 779, 783; vgl. auch Vollmer, DB $1982,1670 \mathrm{ff}$. 
27. These: Zu befürworten ist aber die Festlegung einer Pflicht der Tarifvertragsparteien, im Falle einer Insolvenzsituation über die Anpassung der tariflich festgelegten Entgelte zu verbandeln.

Angemessen erscheint es aber, den Tarifvertragsparteien die Pflicht aufzuerlegen, hinsichtlich eines im Sanierungsverfahren befindlichen Unternehmens über die Anpassung der tariflich festgelegten Entgelte jedenfalls zu verbandeln. Eine solche Verpflichtung würde sie besonders auf ihre Verantwortung hinweisen und könnte zugleich den Gewerkschaften ihre Position gegenüber den Mitgliedern erleichtern. 